\title{
The motion of vibrating systems in Schwarzchild spacetime
}

\author{
A. Hees ${ }^{1}$, L. Bergamin ${ }^{2}$ and P. Delva ${ }^{3}$ \\ ${ }^{1,2,3}$ European Space Agency, The Advanced Concepts Team \\ Keplerlaan 1, 2201 AZ Noordwijk, The Netherlands \\ ${ }^{1}$ Royal Observatory of Belgium (ROB) \\ Avenue Circulaire 3, 1180 Bruxelles, Belgium \\ ${ }^{1}$ aurelien.hees@oma.be ${ }^{2}$ bergamin@tph.tuwien.ac.at ${ }^{3}$ Pacome.Delva@esa.int
}

\begin{abstract}
In this communication, the effects of vibrations at high frequencies onto a freely falling two-body system in Schwarzschild spacetime are investigated. We present these effects for different kinds of reference motions, all of which are placed in regions of weak gravitation: circular orbits, radial free fall (with different initial velocities) and radial free fall with a small tangential velocity. The vibrations induce a change in the motion of the vibrating system, which is characterized by a radial deviation between the vibrating system and the reference motion of the non-vibrating system. For a circular orbit, we show that the maximal radial deviation increases linearly with the initial radius. For a radial free fall, the radial deviation after one oscillation decreases quadratically with respect to the initial radius.
\end{abstract}

Keywords. gravitation, general relativity, Schwarzchild geometry, vibrating systems

\section{Introduction}

Changing the shape of a free-falling body in a gravitational field can induce a change in its motion. In Newtonian physics, such effects are well known and could be used in space technology for propellentless propulsion as presented in Martinez et al. (1987). These proposals are based on resonant effects, where the change of shape is linked to the motion of the body in space. More recently, similar situations have been studied in the framework of general relativity (see for example Wisdom (2003), Guéron et al. (2006), Avron and Kenneth (2006) , Guéron and Mosna (2007)) where non-resonant effects can become large.

In the present communication, we consider the free fall of an oscillating two-body system around a central body, where the deviation of the motion of a vibrating system with respect to the motion of the same system without vibration is investigated. Three different classes of initial conditions (or reference motions) will be presented: a circular orbit (described in Bergamin et al. (2009b)), a radial free fall (described in Guéron and Mosna (2007), Bergamin et al. (2009a)) and a radial free-fall with a tangential initial velocity. We will show that in general relativity new interesting effects appear even in regions of weak gravitation, which are not present in Newtonian physics, and we will describe how these effects depend on the initial conditions.

\section{The model}

A vibrating or oscillating system is implemented as a collection of point masses whose relative positions are related by time dependant constraints. The model used here is described in Guéron and Mosna (2007), Bergamin et al. (2009a), Bergamin et al. (2009b): 
two point masses connected by a massless tether, whose length $l(t)$ is imposed by an oscillating constraint. The constraint is described by four parameters: its frequency $\omega$, its amplitude $\delta_{l}$, its minimum length $l_{0}$ and an asymmetry parameter $\alpha$ (it describes how much the oscillation fails to be symmetric in time). The vibrating system evolves in the surrounding of a central body described by a Schwarzschild spacetime. We implement the oscillating constraint in Schwarzschild coordinates. We do not describe a particular system, such as a molecule or a crystal. To do so, one would have to describe this particular system in the Fermi-Walker coordinates of the reference motion, which are in good approximation comoving coordinates of the vibrating system. The system dynamics is studied in the orbital plane: each body is described in terms of two variables $\left(r_{i}, \phi_{i}\right)$ (where $\left.i=1,2\right)$, and we define the geometrical center of the system whose radial coordinates is $r=\left(r_{1}+r_{2}\right) / 2$.

The equations of motion of the vibrating system are derived from the action

$$
S=-\int \mathrm{d} t\left[\sqrt{L_{1}}+\sqrt{L_{2}}+\lambda\left(r_{2}-r_{1}-\sqrt{l^{2}(t)-r_{1}^{2} \theta^{2}}\right)\right],
$$

where $\lambda$ is a Lagrange multiplier, $t$ is the Schwarzschild time coordinate, $l(t)$ is the length of the constraint, $\theta=\phi_{2}-\phi_{1}$, and

$$
L_{i}=\left(1-\frac{r_{s}}{r_{i}}\right)-\left(1-\frac{r_{s}}{r_{i}}\right)^{-1} \dot{r}_{i}^{2}-r_{i}^{2} \dot{\varphi}_{i}^{2}
$$

where $\left(\dot{)} \equiv \mathrm{d} / \mathrm{d} t\right.$ and $r_{s}$ is the Schwarzschild radius. In addition to these equations of motion, one has to choose initial conditions. Different initial conditions will be presented here: an initial condition leading to a circular orbit, different initial conditions for a radial free fall and some intermediate cases.

\section{Circular orbit}

For the first set of initial conditions we choose vanishing radial velocity $\dot{r}(t=0)=0$ with $r(t=0)=r_{0}$, while $\phi_{i}(t=0)=0$ and $\dot{\phi}_{i}(t=0)$ is determined in such a way that the reference motion (for the non-vibrating system) is a circular orbit. This situation is thoroughly discussed in Bergamin et al. (2009b). Here, the deviation of the motion of the vibrating system with respect to the motion of the non-vibrating one is characterized by two effects: a radial deviation and an oscillation of the system around the radial axis. The radial deviation implies that the trajectory becomes a quasi-elliptical orbit. It is possible to quantify the maximal radial deviation $\Delta r$ between the vibrating system and the non-vibrating one. The analytical expansion in Bergamin et al. (2009b) leads to

$$
\begin{aligned}
\frac{\Delta r}{r_{0}}= & \frac{\delta_{l}^{2} \omega^{2}}{c^{2}} \frac{S_{1}(\alpha)}{\left(1-\frac{r_{s}}{r_{0}}\right)\left(1-\frac{3 r_{s}}{r_{0}}\right)}+\frac{5}{2} \frac{\delta_{l}}{r_{0}} \frac{1-\frac{9 r_{s}}{r_{0}}}{1-\frac{3 r_{s}}{r_{0}}}\left(2 \frac{l_{0}}{r_{0}} S_{2}(\alpha)+\frac{\delta_{l}}{r_{0}} S_{3}(\alpha)\right) \\
+ & \mathcal{O}\left(\frac{i^{2}}{c^{2}} \frac{l^{2}}{r_{0}^{2}}\right)+\mathcal{O}\left(\frac{i^{4}}{c^{4}}\right)+\mathcal{O}\left(\frac{l^{4}}{r_{0}^{4}}\right)
\end{aligned}
$$

where $c$ is the speed of light and $S_{i}(\alpha)$ are some functions in $\alpha$ of the order one. The first term of this expansion is a purely relativistic effect not present in Newtonian theory and it grows linearly with the initial radius $r_{0}$. The second term is present in Newtonian theory (with $r_{s} / r_{0} \rightarrow 0$ ) and it decreases as $1 / r_{0}$. Then the difference between general relativity and the Newtonian theory of gravitation increases linearly with the initial radius. This analytical result is confirmed by numerical simulations as can be seen in Figure 1 (a). 


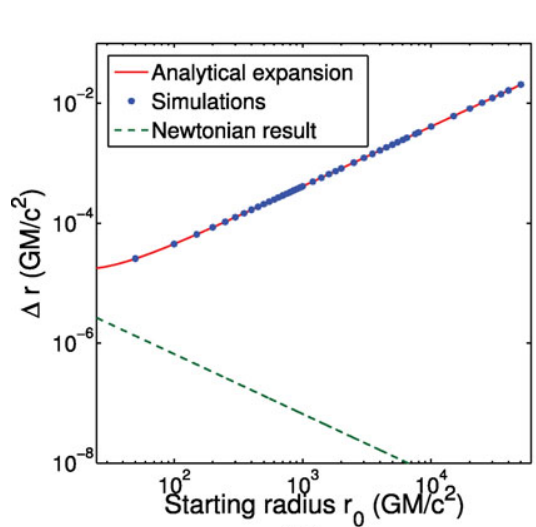

(a)

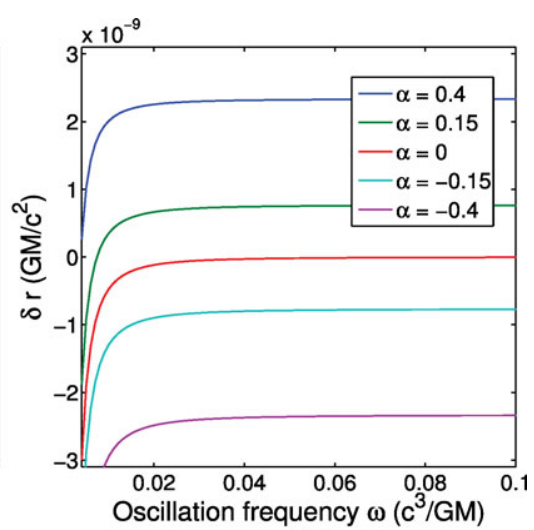

(b)

Figure 1. (a): Maximal radial deviation in case of a circular orbit as a function of the starting radius $r_{0}$ for $\omega=0.05$ : comparison of numerical simulations and an analytical expansion. Additionally, the leading classical gravitational term has been included in the figure to illustrate the difference between general relativity and Newtonian gravity. (b): Radial deviation after one oscillation period, in case of a radial free-fall with a vanishing initial velocity as a function of the oscillation frequency, for different values of the asymmetry parameter $\alpha$ and for $r_{0}=120 \mathrm{GM} / \mathrm{c}^{2}$.

In summary, for a circular orbit the relativistic radial deviation increases linearly with the radius, increases quadratically with the vibration frequency $\omega$ and the oscillation amplitude $\delta_{l}$, while the effect of the asymmetry parameter $\alpha$ is quite small.

\section{Radial free fall}

Now, we consider the same system in a radial free fall towards a central body. This situation is studied in Guéron and Mosna (2007), Bergamin et al. (2009a). The initial conditions are $\phi_{i}(t=0)=\dot{\phi}_{i}(t=0)=0, r(t=0)=r_{0}$ and $\dot{r}(t=0)=v_{0}$. The case with $v_{0}=0$ was discussed by Guéron and Mosna (2007). In that case, it is possible to slow down or to accelerate the free fall of a system depending on the parameter $\alpha$. Guéron showed that after one oscillation period, the radial deviation is given by

$$
\delta r \approx \Gamma_{\alpha} \frac{\delta_{l}^{2}}{r_{0}^{2}} \frac{G M}{c^{2}},
$$

where $M$ is the mass of the central body, $G$ the gravitational constant and $\Gamma_{\alpha}$ is a factor associated with the asymmetry parameter. This radial deviation again is a purely relativistic effect and it decreases quadratically with the initial radius. Here the asymmetry parameter is very important since $\delta r$ vanishes for $\alpha=0$, is positive (it corresponds to a deceleration) for positive values of $\alpha$ and negative (it corresponds to an acceleration) for negative values of $\alpha$, as can be seen in Figure 1 (b).

Now, we extend the particular initial condition $v_{0}=0$ studied by Guéron and Mosna (2007) to non-vanishing initial velocities. In Figure 2 (a), the radial deviations for different initial velocities $\dot{r}(t=0)=v_{0}$ for a positive value of $\alpha$ are presented. As can be seen, the effect increases if the initial velocity is negative (the system already falls down at $t=0$ ); while in the opposite case (the system is thrown up at $t=0$ ), the effect decreases to become negative at higher velocity. This behavior is certainly not surprising, since the work performed by the oscillations is expected to change sign when the system moves upwards. 

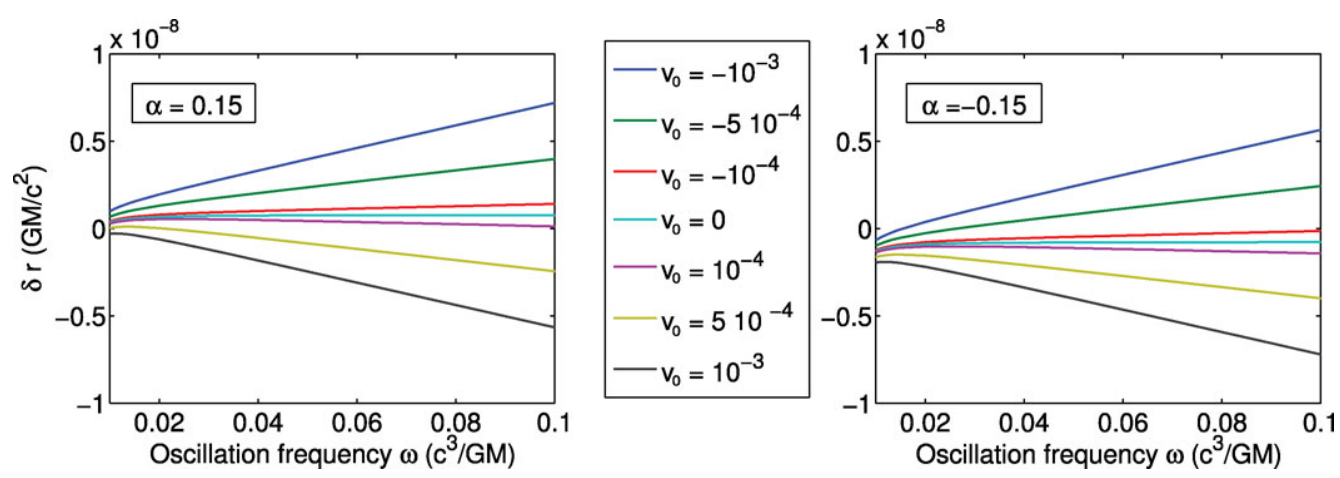

Figure 2. Radial deviation $\delta r$ in the case of a radial free fall, as a function of the oscillation frequency $(\omega)$, for different initial radial velocities $\dot{r}(t=0)=v_{0}$ (expressed as a fraction of $c$ ). In these examples, $r_{0}=120 \mathrm{GM} / \mathrm{c}^{2}$ and (a): asymmetry parameter $\alpha=0.15$. (b): asymmetry parameter $\alpha=-0.15$.

Still, changing the sign of the asymmetry parameter for $v_{0} \geqslant 0$ is not an option to obtain a positive displacement (to slow down the free fall). This situation is depicted in Figure 2 (b), where different initial velocities with negative values of $\alpha$ are presented. The change in the sign of $\alpha$ induces a vertical shift of all curves. Thus, a positive effect is only achievable for a system falling down at high velocities $\left(v_{0} \gg 0\right)$, while for initial conditions $v_{0}=0$ a negative value results over the whole frequency range as seen in Figure 1 (b).

In summary, for a radial free fall, the relativistic radial deviation decreases quadratically with the initial radius, has a plateau in the frequency and the effect of the asymmetry parameter $\alpha$ is important (no effect is found for a symmetric constraint). The radial deviation $\delta r$ is increased if the initial radial velocity is negative (the system is already falling down $v_{0}<0$ ) and is decreased in the opposite case.

\section{Radial free fall with angular velocity}

We have presented the effects of vibrations on a circular orbit and on a radial free fall with different initial conditions. Now, we will study the intermediate case: the deviation of a vibrating system falling down towards a central body with a small angular velocity. The initial angular velocity is expressed as a fraction of the angular velocity of the reference circular orbit, $\dot{\phi}_{i}(t=0)=\beta \dot{\phi}_{c}$, where $\beta=0$ implies a radial free fall and $\beta=1$ a circular orbit. The remaining initial conditions are $\phi_{i}(t=0)=0, r(t=0)=r_{0}$ and $\dot{r}(t=0)=0$. Figure 3 shows the behavior of the radial deviation $\delta r$ for different initial angular velocities. It can be seen that the effect $(\delta r)$ decreases if the angular velocity is increased and becomes negative at high $\beta$ values. Still, the radial relativistic displacement $\delta r$ is not very sensible to the initial $\beta$ conditions as long as the angular velocity remains small. This shows that the vibrations indeed can be used for "gliding".

Similar conclusions apply for negative values of $\alpha$ but since for these values, $\delta r \leqslant 0$ at $\beta=0$, we do not reproduce these cases explicitly.

In summary, a small angular velocity has a very small influence on the relativistic radial deviation $\delta r$ and for high angular velocity, the system will orbit around the central mass. 


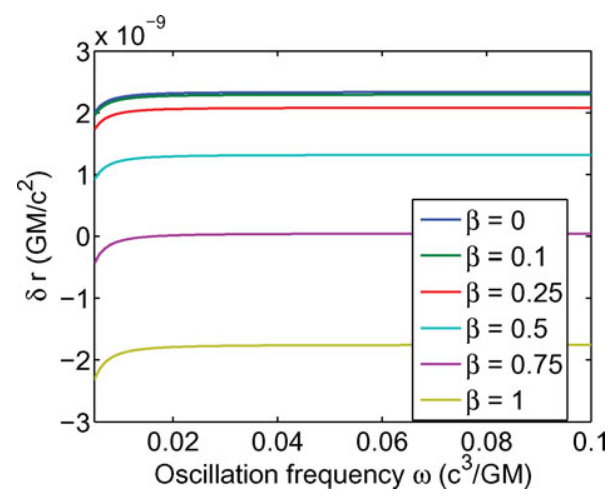

Figure 3. Radial deviation $\delta r$ as a function of the oscillation frequency $\omega$ in case of a radial free fall, with different value of initial angular velocity, expressed as a fraction $\beta$ of the circular angular velocity. In this example, $r_{0}=120 \mathrm{GM} / \mathrm{c}^{2}$ and $\alpha=0.4$

\section{Conclusion}

In this communication we have discussed the influence of oscillations onto the trajectory of a small system in Schwarzschild spacetime in weak gravity field. We have shown that a systematic expansion of these effects in Schwarzschild coordinates can be dominated by a term not present in Newtonian theory of gravitation even in weak gravity field. These effects manifest themselves as a radial deviation from the reference motion and depend on the initial conditions of the system. In particular, the differences between a radial free fall and a circular orbit have been worked out. For the circular case, the maximal radial deviation is increasing linearly with the starting radius, whereas, for the radial free fall, the radial deviation after one oscillation decreases quadratically. Hence, the circular case could be more interesting for experimental verifications. The second difference is the role played by the asymmetry parameter of the vibration. For the circular case, it has a minor impact on the result, while in the radial free fall, it is crucial in order to get a relativistic effect.

\section{Acknowledgments}

The authors would like to thank D. Izzo for important discussions on the topic. A. Hees is a research fellow from FRS-FNRS (Belgian Fund for Scientific Research) for his PhD thesis at ROB-UCL (Université Catholique de Louvain, Belgium) and he acknowledges a FNRS and a NSF grant to attend to the IAU 261 symposium.

\section{References}

Martinez-Sanchez, M. \& Gavit., S. A., 1987, J. Guid. Control Dyn. 10-233

Wisdom, J. 2003, Science 299-2865.

Gueron, E., Maia, C. A. S., \& Matsas, G. E. A. 2006, Phys. Rev. D 73/024020

Avron, J. E. \& Kenneth., O. 2006, New J. Phys., 8-68

Guéron, E. \& Mosna, R. A. 2007, Phys. Rev. D, 75/081501

Bergamin, L., Delva, P., \& Hees, A. 2009 (a), arXiv: gr-qc/0901.2298

Bergamin, L., Delva, P., \& Hees, A. 2009 (b), Class. Quantum Grav., 26/185006 